\title{
Comparación entre las técnicas de la gota de agua y la tijera de Metzembaum en la técnica del TOT (trans obturatriz tape) para la aplicación de la tensión Sling en el ángulo sub-uretral para la cura de la incontinencia urinaria de la mujer en el Hospital Nacional Arzobispo Loayza, Lima-Perú
}

\author{
Alejandro Siu ${ }^{1}$, Diego Siu²
}

\section{RESUMEN}

Objetivo: comparar dos técnicas que se usan en las cirugías donde se utiliza la TOT para regular y dar la tensión adecuada al Sling sub-uretral en la cura de la Incontinencia Urinaria de Esfuerzo femenina: la técnica de la gota de agua y la de la tijera de Metzembaum, para evaluar la morbilidad infecciosa de las vías urinarias y el tiempo desarrollado en ej ecutar cada una de ellas.

Material y Métodos: El estudio, se realizó en el Servicio de Ginecología del Hospital Nacional Arzobispo Loayza, entre noviembre del 2009 y setiembre del 2011, aleatoriamente en dos grupos de pacientes: grupo "A", que utilizó la técnica de la gota de agua y el "B”, que utilizó la Tijera de Metzembaum, siendo un clásico estudio prospectivo analítico observacional de casos y controles.

Resultados: La morbilidad infecciosa fue prácticamente la misma, pero si existió una diferencia sustancial de tiempo operatorio a favor del grupo "B".

Conclusión: Ambas técnicas utilizadas en el TOT fueron efectivas y solo se diferenciaron por el menor tiempo operatorio con la tijera de Metzembaum (Horiz Med 2014; 14(3): 28-32)

Palabras clave: Incontinencia urinaria de esfuerzo, TOT, procedimientos quirúrgicos urológicos. (Fuente: DeCS BIREME).

Comparison between two different techniques, the drop of water technique versus the Metzembaum scissors technique, to give an adecuate tension to the suburethral sling where TOT is used as treatment for urinary incontinence in The Arzobispo Loayza national hospital, Lima- Peru

\section{ABSTRACT}

Objective: The purpose of this prospective study was to compare two different techniques, the drop of water technique versus the Metzembaum scissors technique, to give an adequate tension to the suburethral sling where TOT is used as treatment for urinary incontinence.

Material and Methods: The study took place in the Gynecology service of the Arzobispo Loayza National Hospital, from November 2009 to September 2011. The two groups of patients; Group "A" (Drop of water technique) and Group "B" (Metzembaum scissors technique) were randomly chosen.

Results: It was shown thatmorbidity caused by infection was similar in both groups, nevertheless, a substantial reduction in surgery time existed in Group "B".

Conclusion: Both techniques used in the TOT were effective and only differentiated by the shorter surgery time with Metzembaim scissors. (Horiz Med 2014; 14(3): 28-32)

Key words: Stress urinary incontinence, TOT surgical procedure (Source: MeSH NLM).

1 Doctor en Medicina, Ginecólogo Obstetra, Profesor Principal de la Universidad Peruana Cayetano Heredia, Ex-Jefe de Departamento de Ginecología y Obstetricia y del Servicio de Ginecología del Hospital Nacional Arzobispo Loayza.

2 Interno de Medicina de la Universidad Peruana Cayetano Heredia. 
Comparación entre las técnicas de la gota de agua y la tijera de Metzembaum en la técnica del TOT (trans obturatriz tape) para la aplicación de la tensión Sling en el ángulo sub-uretral para la cura de la incontinencia urinaria de la mujer en el Hospital Nacional Arzobispo Loayza, Lima-Perú

\section{INTRODUCCIÓN}

En 1966, con el reporte de Ulmstein en Europa, informando sobre la Técnica de la Tensión Free Vaginal Tape - TVT - colocando un Sling para el tratamiento quirúrgico de la incontinencia urinaria de esfuerzo femenina, se inicia la revolución en el manejo de esta entidad. Constituyéndose a fines de los años 90, como el procedimiento más usado en el mundo, habiéndose realizado más de 500,000 cirugías de ese tipo con tasas de curación a los $1,3,5$ y 7 años de $91 \%, 86 \%, 87 \%$ y $81.3 \%$ respectivamente (1-5).

En la TVT, se realiza el paso ciego de la aguja a través del espacio retropúbico $(6,7)$. En Francia, Delome, presentó en el 2001, una variante de ese procedimiento: el TOT, colocando un Sling subureteral a través del agujero obturador de la pelvis (8), desde entonces. se han realizado miles de cirugías de ese tipo en el mundo $(9,10,11,12$, $13,14)$.

La simpleza de la técnica y la baja secuela de complicaciones, proporcionan a la técnica del TOT, las ventajas de: evitar las incisiones abdominales y el paso retropúbico de la aguja, disminuyendo las lesiones graves de: vejiga, intestino, vasos sanguíneos y nervios, haciendo innecesario el uso rutinario de la cistoscopia intraoperatoria $(13,14$, 15,16).

Desde el 16 de mayo del 2006, se realiza el TOT en nuestra institución (17), y, vemos con satisfacción, que cada vez se hace mej or, la curva de aprendizaj e está en su meseta con una suficiencia adecuada. Regulando la tensión del Sling o Malla suburetral, mediante dos métodos: la técnica de la Gota de Agua y la técnica de la tijera de Metzembaum.

La técnica de la Gota de Agua requiere habilidad y teóricamente implicaría una mayor morbilidad infecciosa por la instrumentación y la prolongación del tiempo operatorio, a diferencia de la técnica de la tijera de Metzembaum que sería más conveniente $(14,17)$.

Por lo que, nos propusimos evaluar ambas técnicas y compararlas en los aspectos de morbilidad infecciosa de las vías urinarias y en el tiempo desarrollado en la ejecución del procedimiento.

\section{MATERIAL Y MÉTODOS}

El estudio se realizó en el Hospital Nacional Arzobispo Loayza, en el Servicio de Ginecología, desde el mes de noviembre del 2009, hasta setiembre del 2011. Se reclutaron a 50 pacientes, quienes fueron sometidas a la TOT con la técnica de la Gota de Agua para la evaluación de la tensión adecuada de ajuste de la uretra, constituyendo el Grupo "A" y a otras 50 pacientes, sometidas a la TOT pero aplicando la técnica de la tijera de Metzembaum, constituyendo el Grupo "B". Siendo un clásico estudio prospectivo, analítico, observacional de casos y controles.

La incorporación a ambos grupos, fue de manera aleatoria y voluntaria desde los Consultorios de Ginecología de la institución siempre que cumplieran con el criterio diagnóstico.

La evaluación post operatoria después del alta de las pacientes, se hizo entre las dos a cuatro semanas después de la intervención.

La técnica quirúrgica de la TOT fue semejante a la referida por Brioso $(14,17)$.

Técnica de la Gota de Agua:

Posicionada la malla en la zona suburetral, se reguló la tensión hasta el punto en que con maniobras de Valsalva excesivas (tos fuerte) no se manifieste la incontinencia urinaria, previamente se instila entre 300 a 400 cc de agua destilada a través de una sonda de Foley a la vejiga vacua. Se recomienda que sólo aparezca el brillo de la gota de suero en el meato uretral ya que al recuperar la posición ortostática y al descender la pared anterior de la vagina, la angulación sobre la uretra actúa como un mecanismo de continencia urinaria. Figura 1.

Técnica de la Tijera de Metzembaum:

No se realiza la maniobra de Valsalva, sólo se deja la malla libre de tensión permitiendo el pasaje holgado de una tijera Metzembaum cerrada entre la malla y la uretra. Figura 2. 


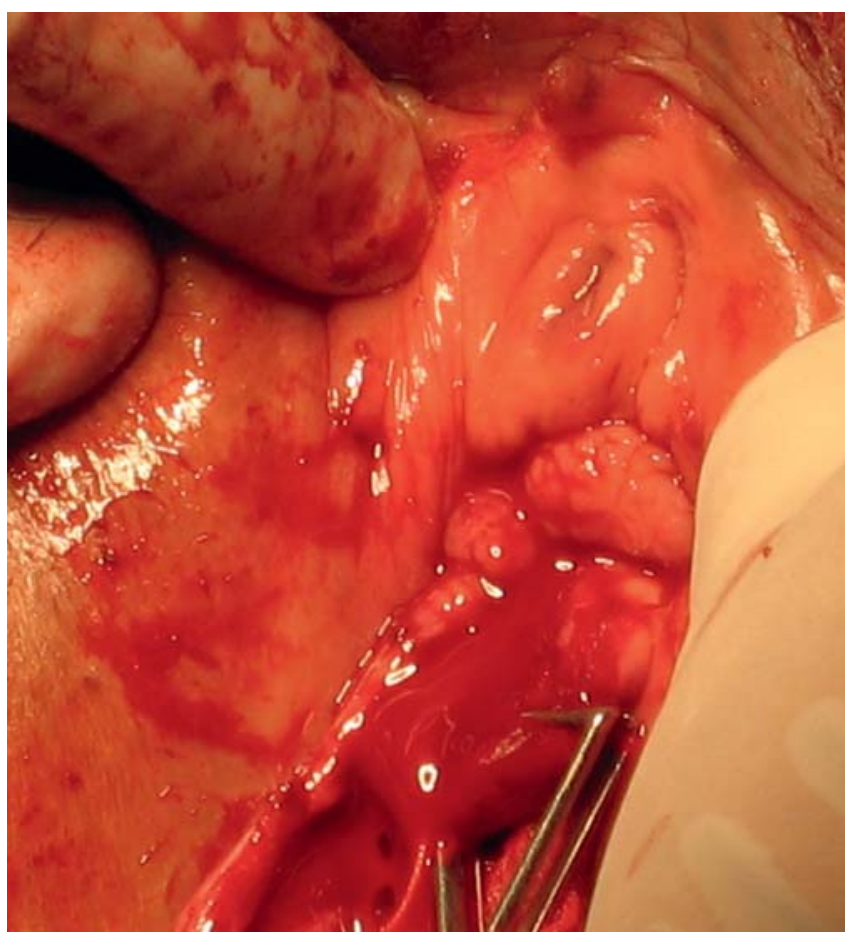

Figura 1. Haciendo pujar a la paciente, hasta observar la gota de agua en el meato uretral

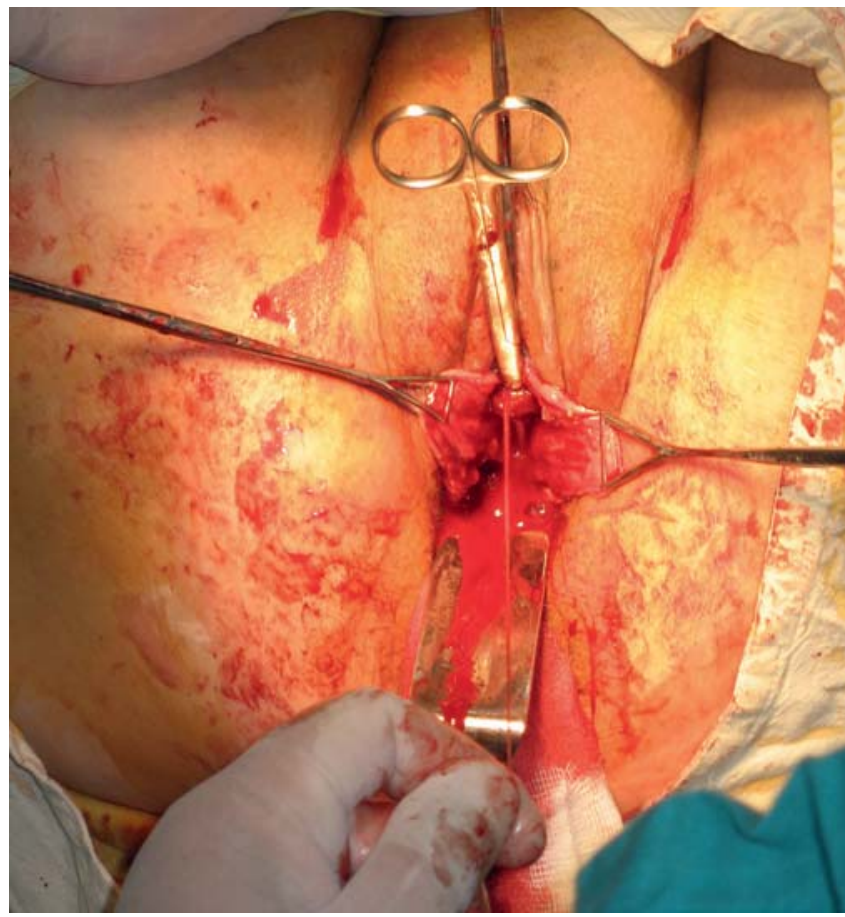

Figura 2. Ajuste de la malla suburetral con la tijera de Metzembaum
En el posoperatorio, se exigió la deambulación precoz.

La Sonda vesical se dejaba abierta por 12 a 24 horas (de acuerdo al tipo de anestesia).

Luego de orinar, si no había tenesmo o sensación disfuncional, se daba el alta. Si había tenesmo o retención urinaria, se medía el residuo y si éste era menor de $100 \mathrm{ml}$, se daba el alta de acuerdo a la cirugía que se acompañó al TOT.

\section{RESULTADOS}

El número de pacientes reclutadas fueron: cincuenta para el Grupo "A" de la técnica de la Gota de Agua y de cincuenta para el Grupo "B" de la Tijera de Metzembaum.

La edad y paridad promedio, fueron semejante para ambos grupos: 57 años y 5 , respectivamente.

Enel Grupo “A” se realizaron 39 (78\%) Histerectomías Vaginales con Colpoperineorrafía antero-posterior, 9 (18\%) Colpoperineorrafías antero-posterior sin Histerectomías Vaginales y 2 (4\%) procedimientos de TOT puros.

Enel Grupo “B”, se realizaron 37 (74\%) Histerectomías Vaginales con Colpoperineorrafía antero-posterior, $9(18 \%)$ Colpoperineorrafías antero-posterior sin Histerectomías Vaginales, 2 (4\%) procedimientos de TOT puros y 2 (4\%) Histerectomías abdominales.

El tiempo promedio para la ejecución de la técnica de la gota de agua en el Grupo "A" fue de 20 minutos y para la técnica de la tijera de Metzembaum en el Grupo "B”, fue de 9 minutos.

Las complicaciones inherentes a la técnica en el Grupo “A”, fueron:

- Retención urinaria en tres pacientes post-retiro de sonda $(6 \%)$

- Infección urinaria en cuatro pacientes (8\%)

- Dehiscencia de sutura de la colpoperineorrafía anterior con exposición de la malla en tres pacientes (6\%)

Las complicaciones inherentes a la técnica en el Grupo “B”, fueron: 
- Retención urinaria en tres pacientes post-retiro de sonda $(6 \%)$

- Infección urinaria en tres pacientes (6\%)

- Dehiscencia de sutura de la colpoperineorrafía anterior con exposición de la malla en dos pacientes $(4 \%)$

Las complicaciones no inherentes a la técnica en el Grupo “A”, fueron:

- Hematoma de cúpula post histerectomía vaginal en dos (4\%) pacientes

- Infección de cúpula post histerectomía vaginal en cuatro (8\%) pacientes

- Enterocolitis en una (2\%) paciente

- Neumonía en una (2\%) paciente.

Las complicaciones no inherentes a la técnica en el Grupo “B”, fueron:

- Hematoma de cúpula post histerectomía vaginal en tres $(6 \%)$ pacientes

- Infección de cúpula post histerectomía vaginal en cuatro (8\%) pacientes

- Hematoma en la Colpoperineorrafía posterior en una (2\%) paciente

- Infección de la herida de pared abdominal en una (2\%) de las Histerectomías abdominales.

\section{DISCUSIÓN}

El tratamiento de la incontinencia urinaria de esfuerzo (IUE), ha ido variando en el tiempo, siendo actualmente, la porción suburetral de la uretra media determinante en cualquier técnica quirúrgica que se utilice para tratar la IUE (18).

La incidencia de la incontinencia urinaria, parece ser mayor en mujeres con mayor paridad y se relaciona con el mayor tiempo postparto por lo que la cesárea, sería un factor protector (19). Lo que evidenciamos en el grupo estudiado, al observar una paridad de 5 . Sin embargo, recientemente se le da más valor al peso del feto, superior a $4 \mathrm{~kg}$, que a la paridad (20).

Para que el esfínter uretral funcione eficazmente es fundamental el soporte anatómico adecuado por inserciones pélvicas extrínsecas. La configuración anatómica que confiere el anclaje de la fascia endo pélvica y la pared vaginal al músculo estriado del elevador del ano y a los huesos de la pelvis a través del arco tendinoso de la fascia pélvica promueve el almacenamiento de la orina y permite la transmisión de la presión del cuello vesical a la uretra (21).

Lo que se busca con el tratamiento quirúrgico es mantener fija la uretra y la unión uterovesical, y debe plantearse si el manejo conservador no ha funcionado o si la paciente no cumple con los mismos (5)

Diversos estudios, han demostrado el valor de la técnica del TOT (Transvaginal Obturatriz Tape) como una técnica efectiva para el tratamiento de la incontinencia urinaria de esfuerzo, asociada o no a patología ginecológica $(8,11,13,14)$.

Siendo conveniente conocer que técnica utilizar para tener mejores resultados con la TOT: técnica de la gota de agua o la de la tijera de Metzembaum.

En este estudio prospectivo, analítico, observacional de casos y controles, observamos por los resultados, que prácticamente, no existió mayor diferencia significativa en cuanto a la morbilidad infecciosa del tracto urinario, ni tampoco en la retención de orina post retiro de la sonda vesical; pero, si una reducción sustancial del tiempo operatorio entre ambas técnicas, siendo el menor tiempo operatorio con la Técnica de la Tijera de Metzembaum,

Por el menor tiempo operatorio empleado con la técnica de la Tijera, creemos que ésta debería ser de elección en la TOT, con la ventaja que ésta, se puede realizar aún con la paciente inconsciente 0 con anestesia general.

En cuanto al resto de las complicaciones en ambos grupos, la frecuencia fue similar a la descrita en la literatura publicada $(14,17)$.

\section{Fuentes de financiamiento}

El estudio ha sido autofinanciado por el autor.

\section{Conflicto de interés}

El autor declara no tener ningún conflicto de interés. 


\section{REFERENCIAS BIBLIOGRÁFICAS}

1. Ulmsten U, Petros P. Intravaginal Slingplasty (IVS): An ambulatory surgical procedure for treatment of female urinary incontinence. Scand J Urol Nephrol 1995; 29: 75-82.

2. Petros $P$, Ulmsten U. An integral Theory of female urinary incontinence. Experimental and clinical considerations. Acta Obstet Gynecol Scand 1990; 69 (Suppl 153).

3. Ulmsten U, Jonson P, Rezapour M. A three-year follow up of tension free vaginal tape for surgical treatment of female stress urinary incontinence. Br J Obstet Gynaecol 1999; 106: 345-350.

4. Haab F, Sananes S, Amarenco G. Results of the tension-free vaginal tape procedure for the treatment of type II stress urinary incontinence at a minimum follow up of a 1 year. J Urol 2001; 165(1): 159-62.

5. Balmforth J, Cardozo L. Trends toward less invasive treatment of female stress urinary incontinence. Urology 2003; 62(4 Suppl): 52 60 .

6. De Lancey J. Structural support of the urethra as it relates to stress urinary incontinence: The hammock hypothesis. Am J Obstet Gynecol 1994; 170: 1713-1723.

7. Walters M, Tulikangas P, LaSala C, Muir T. Vascular injury during tension free vaginal tape procedure for stress urinary incontinence. Obstet Gynecol 2001; 98(5 Pt 2): 957-9.

8. Delorme E. La bandelette trans-obturatrice: un procédé miniinvasif pour traiter l'icontinence urinaire d'effort de la femme. Prog Urol 2001; 11(6):1306-13.

9. Dargent D, Bretones S, George P: Pose d'un ruban sous urétral oblique par voie obturatrice dans le traitement de l'icontinence urinaire fémenine. Gynécol Obstét Fértil 2002; 30: 576-82.

10. Mellier G, Benayed B, Bretones S, Pasquier JC. Surgical urinary incontinence treatment by suburethral mesh: comparison between retropubic approach (TVT) an obturator approach (TOT). Int Urogynecol J Pelvic Floor Dysfunct 2004; 15(4): 227-32.

11. Delorme E, Droupy S, Delmas V. Transobturator tape (Uratape): a new minimally-invasive procedure to treat female urinary incontinence. Eur Urol 2004; 45(2): 203-7.

12. Faúndez E, González E. Cinta vaginal libre de tensión en el tratamiento de la incontinencia de orina femenina. una nueva forma de inserción: a través del agujero obturador (CLT-AO). Rev Chil Obstet Ginecol 2003; 68(5): 355-360.

13. Roa J, Roa B, et. al. Experiencia con swing sub uretral libre de tensión transobturador (TOT) en el tratamiento de la incontinencia urinaria femenina. Rev Chil Obstet Ginecol 2004; 69(4): 294-300.

14. Briozzo L, Vidiella G, et. Al. Tranvaginal Obturatriz Tape (TOT) en el tratamiento de la incontinencia urinaria de esfuerzo femenina. Rev Med Uruguay 2005; 21:130-140.

15. Tayrac R, Deffieux X, Droupy S, Chauveaud-Lambling A, CalvaneseBenamour L, Fernandez $\mathrm{H}$ y cols. A prospective randomized trial comparing tension-free vaginal tape and transobturator suburethraltape for surgical treatment of stress urinary incontinence. Am J Obstet Gynecol 2004; 190(3):602-8.
16. Whiteside J, Walters $M$. Anatomy of the obturator region: relations to a trans-obturator sling. Int Urogynecol J Pelvic Floor Dysfunct. 2004; 15(5):223-26.

17. Siu A, y cols. Técnica de colocación de un sling sub-uretral a través de los agujeros obturatrices (TOT), para el tratamiento de la incontinencia urinaria de esfuerzo femenina. Revista del Viernes Médico 2007; (32):26-31.

18. Alvarez Y, Gutiérrez N, Zubizarreta Y. Transvaginal obturatriz tape en el tratamiento de la incontinencia urinaria de esfuerzo femenina. Rev Ciencias Médicas [online]. 2012, 16(2):164-188.

19. Casey BM, Schaffer JI, Bloom SL, Heartwll SF, McIntire DD, Leveno KJ. Obstetrics antecedents for postpartum pelvis floor dysfunction. Am J Obstet Gynecol 2005; 192: 1655-62.

20. Gorbea-Chávez V, Hernández-Contreras R, Rodríguez-Colorado $S$, Velázquez-Sánchez M, Kunhardt-Rasch IIJ. Factores de riesgo asociados a incontinencia urinaria y anal posterior a evento obstétrico. Perinatología y Reproducción Humana 2011; 25(1): 2328.

21. Donoso 0 , et al. Cinta suburetral transobturatriz (TOT) en la incontinencia urinaria de esfuerzo: continencia a mediano plazo y evolución de los síntomas irritativos vesicales. Rev Chil Obstet Ginecol 2007; 72(6):366-373.

\section{Correspondencia:}

Alejandro Siu Au

Dirección: Av. De las Artes Norte 1488 San Borja. Lima, Perú.

Teléfono: 999661002

Correo electrónico: alejandrosiu@infonegocio.net.pe 